\title{
p-di-pyrrole Benzene Derivatives - A New Class of Highly Active HIV-1 CA Inhibitors
}

\section{Nilanjana Biswas ${ }^{1}$ Sangita Ghosh ${ }^{1}$ and Arijit Bag $^{2,3}$}

${ }^{1}$ Department of Chemistry, Sree Chaitanya College, Habra, West Bengal, India

${ }^{2}$ Department of Chemical Sciences, Indian Institute of Science Education and

Research, Kolkata, West Bengal, India

${ }^{3}$ Department of Applied Sciences, Maulana Abul Kalam Azad University of

Technology, Simhat, Haringhata, West Bengal, India

*Corresponding Author: Arijit Bag, Department of Chemical Sciences, Indian

Institute of Science Education and Research and Department of Applied Sciences,

Maulana Abul Kalam Azad University of Technology, Simhat, Haringhata, West

Bengal, India.

\author{
Received: August 24, 2020 \\ Published: February 27, 2021 \\ (C) All rights are reserved by Arijit Bag., et al.
}

\begin{abstract}
Background: In spite of so many FDA approved HIV drugs, HIV infections and deaths are increasing across the glob. Thus, HIV research is still important and challenging. Finding of low cost highly effective mutation resistant HIV drug is the prime destination of HIV researchers. Small organic molecules may be cost effective if they provide desired drug activities in this regards.

Objective: Finding of small organic molecules with high HIV drug potency is the main target of present research. Since HIV-1 capsid assembly inhibitors (CA) are mutation resistant, we focused on finding of inhibitors of this class.

Methods: Few randomly selected small organic molecules are tested as HIV-1 CA inhibitors through molecular docking using Docking Server. Only effective compounds are used for further calculations to predict $\mathrm{IC}_{50}, \mathrm{CC}_{50}$, LogP. For these cal-culations, Density Functional theory (DFT), Quantum Computation Methodology (QCM), Relative $\mathrm{IC}_{50}$ methodology (RICM) and group contribution methodology for LogP calculation are used.

Results: HIV-1 CA inhibitory capacity of p-di-pyrrole benzene and its derivatives are found very good from the docking methods. It is observed that, among three compounds which are promising, o-ethyl p-di-pyrrole benzene has the highest inhibition constant as an HIV-1 CA inhibitor. Quantum computations of $\mathrm{IC}_{50}, \mathrm{CC}_{50}$ and LogP also supported the docking results. o-ethyl p-di-pyrrole benzene shows desirable activity as HIV-1 CA inhibitor.

Conclusion: Since o-ethyl p-di-pyrrole benzene is a small organic molecule it would be easily synthesizable and cost-effective. Thus, it may be a very good HIV drug. To exaggerate its prospect we have also studied and reported different important properties of these compounds like $\mathrm{IC}_{50}, \mathrm{CC}_{50}$, LogP, etc., which are required before pre-clinical trials. These results are also in favor of o-ethyl p-di-pyrrole benzene to be a drug in practice.
\end{abstract}

Keywords: HIV-1 Capsid A Inhibitor; $\mathrm{IC}_{50}$; $\mathrm{CC}_{50}$; LogP; DFT; p-di-Pyrrole Benzene

\section{Introduction}

There are more than 25 FDA-approved HIV-1 drugs [1-4] for seven classes; nucleoside reverse transcriptase inhibitors (NRTIs), nucleotide reverse transcriptase inhibitors (NtRTIs), non-nucleoside reverse transcriptase inhibitors (NNRTIs), protease inhibitors (PIs), fusion inhibitors (FIs), co-receptor inhibitors (CRIs) and in- 
tegrase inhibitors (INIs) [5] that target one of the three viral enzymes require for completing its (HIV virus) life cyclein the host body (human body) [6]. There are three key steps involved in viral attack; attachment, replication and assembling [6-9]. HIV virus after entering into the blood circulation system of human body, tries to get attached to CCR5 receptor [10] of CD4 cells [11], which is one of the two T-cell types lymphocytes of white blood cell. CD4 cells which control and direct the immune response and coordinate to other immune cells, act as a virus manufacturing machine after HIV infection. In spite of many avail-able drugs, lifelong therapy is required for sustained viral suppression. Due to very fast mutation of HIV-1 [12,13], drug resistance is acquired within very short period of time. Moreover, cross-resistance [14] within mechanistic classes underscoring the need to discover new class of HIV-1 inhibitor.

HIV-1 capsid (CA) protein plays essential roles in the viral replication process which is an attractive new therapeutic target for antiretroviral treatment [15]. During assembling and budding the proteolytic cleavage of 55-kDa Gag polyprotein yields CA protein which provides key protein- protein interactions among immature virion proteins to assemble into the cone- shaped central capsid that surrounds the viral RNA genome, reverse transcriptase (RT) and integrase (IN) [16]. CA mutations impair the assembly and inhibit viral replication. $\mathrm{N}$-terminal domain of CA (CANTD) includes 1 to 146 residues [17] and C-terminal domain (CACTD) is composed of 151 to 231 residues [18]. These two highly helical domains are connected through a very short (only four residues) flexible linker. High-resolution structures of CA hexagons and pentagons are reported by Pornillos., et al. [1921]. There are three independent inhibitor binding sites on CA; at the junction of helices $1,2,4$ and 7 , a conserved hydrophobic cleft within the CACTD four-helix bundle and between helix 4 and helix 7 of the CANTD [22]. CA binding inhibitors of several classes have been reported and providing evidence that CA may be a viable drug target. Christopher., et al. [23] studied two different classes of capsid inhibitors and found that mutations conferring resistance to both classes of compound had two different types of effects. Substitutions within the inhibitor binding site decrease the inhibitor binding affinity, but at the same time viral fitness also decreases. Thus, though due to mutation inhibitor binding probability decreases, viral replication processes do not enhance. This suggests that the development of drug resistance against capsid assembly inhibitors due to mutation is not pos- sible for the HIV virus. Christopher., et al. also shown that due to mutation, stability of inhibitor-enzyme complex increases. Thus, possibility of the development of drug resistance against capsid assembly inhibitors is very less for those inhibitors whose protein binding free energies are highly negative.

In spite of enormous effort, highly efficient HIV Capdi-A inhibitor drug is not market available, though, different groups around the world are continuously working in this field and prospective results are reported in a rapid basis [24-26]. At present, sensational progress in this field is achieved, but, the unavailability of drugs is mostly due to the cost effectiveness of the product reported. Thus, we model very small organic molecules which should be synthetically cost effective and at the same time highly active. For this purpose we systematically study HIV Capsid-A inhibitor capacity of different isomers of di-pyrrole benzene and its alkyl and halide derivatives. For geometry optimization we use Density Functional Theory (DFT) calculation with the help of GAUSSIAN 09 package. Molecular docking method is used for binding affinity calculation using docking server.

\section{Materials and Methods}

Materials preparation

The aim of this work is to find out small organic com-pounds which will be active as HIV-1 CA inhibitor. Thus, we started to compute the bio-activity of small organic com-pounds using molinspiration [27] which predict the bio-activity from quantitative structure-property relationships (QSPR) calculatio [28]. This program package works on-line. We have studied few such small molecules up to a maximum of three substitutions, systematically. Among nearly thirty odd samples, we have chosen only six samples for our docking study which showed better bio-activity among these compounds. After docking we have chosen only three compounds for Density Functional Theory (DFT) study which has $\mathrm{IC}_{50}$ in the micro molar rang. Only these compounds are reported here.

Methods used for different calculations

Molecular Docking method

Molecular docking methods [29-31] are well known and used globally for protein ligand interaction study. Molecular docking uses a classical force field for energy calculation. By the energy minimization search we determine the most probable binding site of a protein. The binding constant is also countable 
from docking. In the present study, Docking Server [32] is used for the docking calculation. This also works on-line. We used only the free accessed version. Different docking parameters which are used for our calculation is presented in table 1.

\begin{tabular}{|l|c|}
\hline \multicolumn{1}{|c|}{ Parameters } & Value \\
\hline tstep & 0.2 \\
\hline qstep & 5.0 \\
\hline dstep & 5.0 \\
\hline rmstol & 2.0 \\
\hline ga-pop-size & 150 \\
\hline ga-num-evals & 250000 \\
\hline ga-num-generations & 540000 \\
\hline ga-run & 10 \\
\hline
\end{tabular}

Table 1: Docking parameters used.

Gasteiger partial charges were added to the ligand atoms. Non-polar hydrogen atoms were merged, and rotated bonds were defined. Docking calculations were carried out on di- pyben protein model. Essential hydrogen atoms, Kollman united atom type charges, and solvation parameters were added with the aid of AutoDock tools. Affinity (grid) maps of xx Å grid points and $0.375 \AA$ spacing were generated using the Auto grid program. AutoDock parameter set- and distance-dependent dielectric functions were used in the calculation of the van der Waals and the electrostatic terms, respectively. Docking simulations were performed using the Lamarckian genetic algorithm (LGA) and the Solis \& Wets local search method. Initial position, orientation, and torsions of the ligand molecules were set randomly. All rotated torsions were released during docking. Each docking experiment was derived from 10 different runs that were set to terminate after a maximum of 250000 energy evaluations. The population size was set to 150 . During the search, a translational step of $0.2 \AA$, and quaternion and torsion steps of 5 were applied.

Quantum IC ${ }_{50}$ Computation Method (QICM)

For IC $_{50}$ computation of our modeled compounds using DFT, we pursue two different methodologies, Quantum $\mathrm{IC}_{50}$ Computation Method (QICM) and Relative IC $_{50}$ Computation Method (RICM) which are recently developed by Bag [33], Bag and Ghorai [34]. QICM is based on the basic en-zyme ligand reaction kinetics proposed by L. Michaelis and M. Menten. From Michaelis-Menten equation we get

$$
I C_{50}=\frac{1}{K_{i}}+\frac{K_{s}}{K_{i}}[S]
$$

Where, $\mathrm{K}_{\mathrm{i}}$ and $\mathrm{K}_{\mathrm{S}}$ are binding constant of inhibitor and substrate respectively. [S] is the substrate concentration. If we replace binding constants by binding free energy we shall get

$$
I C_{50}=\Theta e^{\frac{\Delta G_{i}^{0}}{R T}}
$$

Where,

$$
\Theta=1+[S] e^{\frac{-\Delta G_{s}^{0}}{R T}}
$$

$\Delta \mathrm{G}^{0}{ }_{\mathrm{i}}$ and $\Delta \mathrm{G}^{0}{ }_{\mathrm{s}}$ are the standard Gibbs free energy change for inhibitor and substrate binding with the enzyme, respectively. Thus, $\Theta$ is a process specific constant. We have to evaluate it once for a particular process. To reduce the computation cost, another modification of this method was done and shown effective, accurate and applicable. In this method, $\left(\Delta \mathrm{G}_{\mathrm{i}}^{0}\right) / \mathrm{RT}$ is modeled as

$$
\frac{\Delta G_{i}^{0}}{R T}=B \mu^{2} \omega \mu^{*}
$$

Here, B is a constant which depends on temperature of the enzymatic process and other external conditions which influence the process. $\mu$ and $\mu^{*}$ are dipole moment and hydrophobicity of the inhibitor, respectively. $\omega$ is the reactivity index [35] of the inhibitor.

Relative IC $_{50}$ computation method (RICM)

To reduce the computation cost and increase the implementation territory, an alternative methodology (RICM) to QICM is developed by Bag very recently which is used for the present calculation. Thus, RICM is described in brief here. In RICM, a relative $\mathrm{IC}_{50}$ of an inhibitor is calculated using the $\mathrm{IC}_{50}$ value of a similar inhibitor for a particular process. Thus, the results depend on the choice of the reference compound. But, this methodology is very simple and able to produce very good results as accurate as the QCM method [33]. The equation for computation of IC ${ }_{50}$ using this method is

$$
\left(I C_{50}\right)_{c o m p}=\left(I C_{50}\right)_{r e f} \times e^{\left(\mu_{r e f}-\mu_{c o m p}\right)}
$$

\section{DFT Computational Method}

In DFT method [36-38], the energy of a system is described in terms of the electron density in space. The electron density 
is described by a functional. There are several functional developed so far and still developing. Few functional which are frequently used by the most computational chemistry users, are B3LYP [39], B3PW91 [40], etc. Several market available software included DFT calculations for different purposes. Gaussian [41], GAMESS [42], Q-chem [43], Quantum Expresso [44], VASP [45], NWChem [46], etc. are the leading software in this regards. In general, first, the geometry of a compound is optimized unless exact geometry is available from the experiment. With the optimized geometry different properties of a molecule is computed. To accomplish our present study, we first optimized the structures of our compounds using Gaussian 09 package. These optimized geometries are used to compute dipole moment and the orbital energies which are required to calculate reactivity descriptors.

All geometry optimizations of our used compounds reported here are done following standard procedures taking convergence threshold as 0.000015 Hartree/Bohr. Calculations are performed within the density functional theory (DFT) approximation taking B3LYP functional.

\section{Results}

Three compounds which are found as active HIV-1 CA inhibitors after molecular docking calculations are 1-[4(1H- pyrrol-1-yl) phenyl]-1H-pyrrole (ligand-1), 1,4-dicyclopentyl- 2-methylbenzene (ligand-2) and 1,4-dicy-clopentyl-2ethylbenzene (ligand-3). We first chose pyrrole as substitution because in guanine, pyrrole structure exists. We computed binding affinity of this ligand (from here and onwards, it would be termed as ligand-1) with p24 HIV Capsid-A protein (PBD ID is 1e6j). Then we systematically substitute methyl, ethyl, iodine, chlorine and hydroxyl group to our ligand to search for improvement in binding affinity. Unfortunately, no significant improvement was observed. Thus, for getting better activity we used cyclopentyl functional group as main substitution instead of pyrole.We observed good activity with this substitution. This was followed by methyl and ethyl substitution at the benzene ring ortho to a cyclopentyl group. Docking and DFT results of these two compounds are reported here.

\section{Docking results of Ligand-1 with HIV-1 CA protein}

Docked structure of ligand-1 with HIV-1 capsid-A protein is presented at figure 1. It is observed that as our ligand is small it easily goes to the binding site. But it is too small to tightly fit to the binding cavity. As a result, binding energy is very small, only $-4.9 \mathrm{kcal} / \mathrm{mol}$. There is only one hydrogen bonding formation is observed which is presented in figure 2 . This hydrogen bonding is made by a glutamate residue which is $155^{\text {th }}$ residue. All other interactions are Van der Waals interaction. Though its binding energy is small, its binding frequency is very high, as high as $30.0 \%$. It is because its size is very small and its charge density is very high. It has three interaction zones which is shown in $\mathrm{HB}$ plot presented in figure 3. Estimated inhibition constant for this ligand is $250 \mu$ mole.

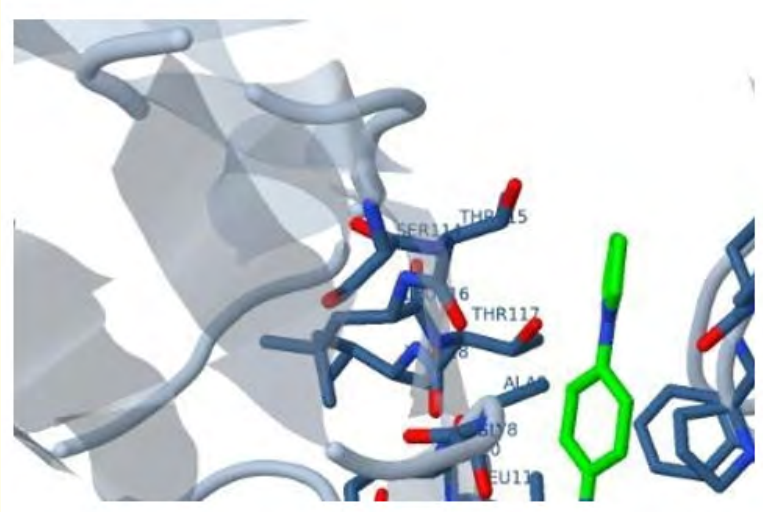

Figure 1: Docked structure of ligand-1 with p24 HIV protein.

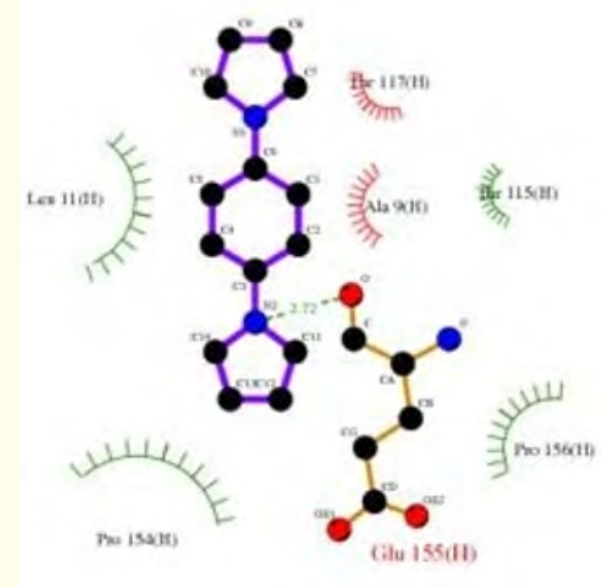

Figure 2: Interacting residues of p24 HIV protein when docked with ligand-1. 


\section{Docking results of Ligand-2 with HIV-1 CA protein}

Docked structure of ligand-2 is presented in figure 4 and interaction plot is presented in figure 5 . Binding affinity of ligand- 2 is $-5.13 \mathrm{kcal} / \mathrm{mol}$ and binding frequency is $50.0 \%$ which is very high. Thus, ligand-2 is an improved inhibitor. It is observed that ligand- 2 is attached to one side of the cavity. Thus, its binding frequency is very high. Inhibition constant of this ligand is $174.9 \mu$ mole.

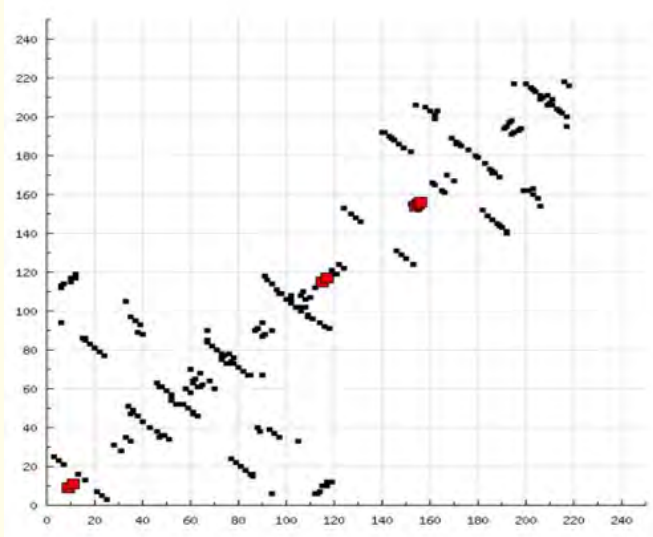

Figure 3: HB plot of ligand-1 with p24 HIV protein.

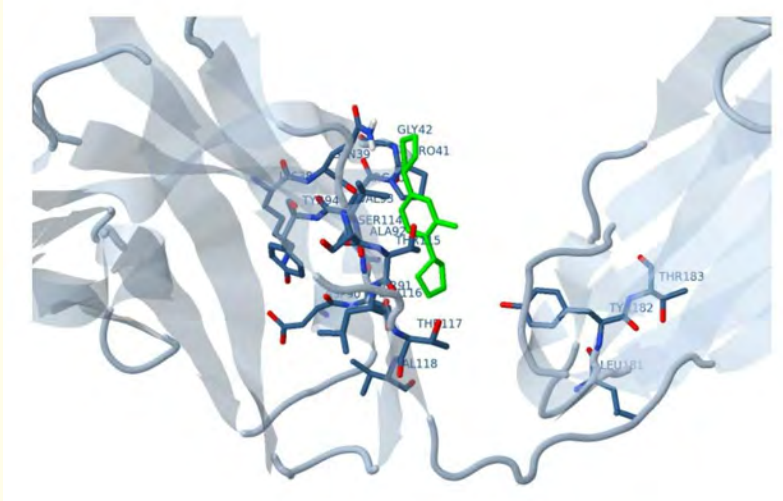

Figure 4: Docked structure of ligand-2 with p24 HIV protein.

Docking results of Ligand-3 with HIV-1 CA protein

We observed that ligand-2 is an improved ligand as a HIV-1 capsid inhibitor compare to ligand-1. Thus, for further improve- ment we substitute with ethyl at the ortho position of benzene ring (ligand-3). Docked structure of ligand-3 is presented in figure 6 and interaction is plotted in figure 7. Binding affinity of ligand-3 is $-6.17 \mathrm{kcal} / \mathrm{mol}$ and binding frequency is $20.0 \%$ which is not high as its methyl counter part but it is acceptable. Its binding frequency is less due to its larger size. It is observed that ligand-3 is attached to the centre of the cavity. Inhibition constant of ligand-3 is only $29.78 \mu$ mole, which is excellent and comparable to market available drugs. Thus, this ligand may be a new drug for HIV-1 capsid-A inhibitor. But for that, this has to pass biological tests.
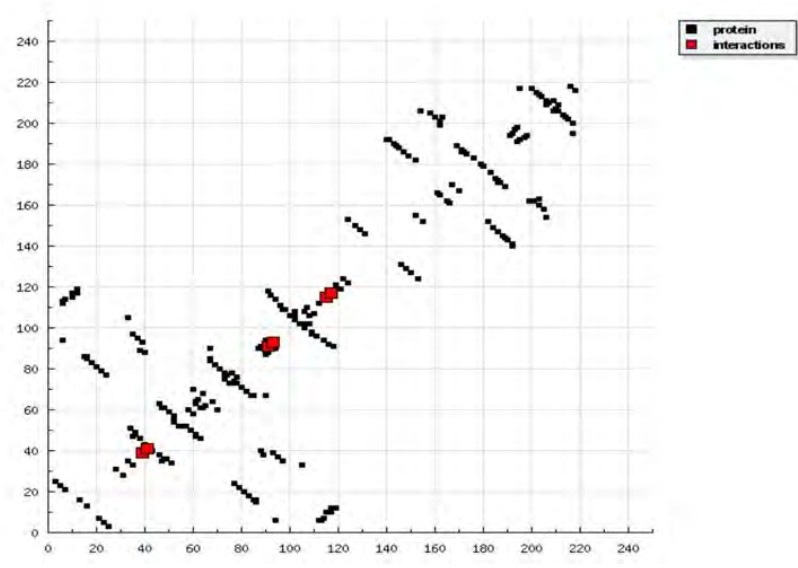

Figure 5: HB plot of ligand-2 with p24 HIV protein.

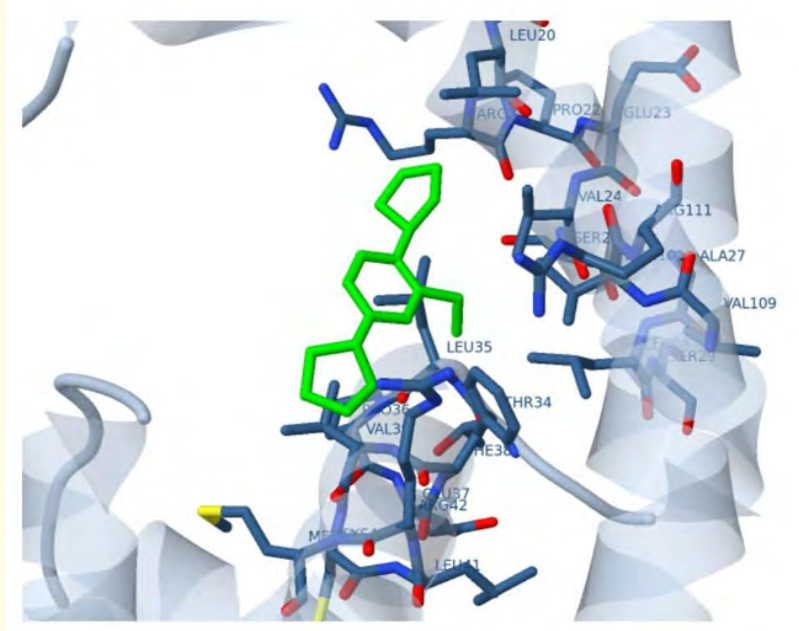

Figure 6: Docked structure of ligand-3 with p24 HIV protein. 
DFT results: $\mathrm{IC}_{50}, \mathrm{CC}_{50}$ and $\mathrm{LogP}$

From the docking study we observed that three p-di-pyrrole benzene derivatives show good activity as HIV-1 Capsid inhibitor. Thus, we compute $\mathrm{IC}_{50}, \mathrm{CC}_{50}$ and LogP of these compounds. For this purpose, we optimized these compounds using Gaussian 09 . The optimized geometry of o-ethyl p-di-pyrrole benzene is presented in figure 8 . We used two different methods of $\mathrm{IC}_{50}$ computation, quantum computation method ( Q I C M) developed by Bag and Ghorai [34] and relative IC $_{50}$ computation method (RICM) proposed by Bag [33]. LogP is computed using on-line platform of molinspiration which has very good accuracy [47]. Toxicity of these compounds $\left(\mathrm{CC}_{50}\right)$, are also computed using relative toxicity method prescribed by Bag and Ghorai [35]. Computed results are presented in table 2 .

\begin{tabular}{|l|c|c|c|c|}
\hline Compounds & $\begin{array}{c}\text { Dipole } \\
\text { moment (D) }\end{array}$ & Log P & $\begin{array}{c}\mathbf{I C}_{\mathbf{5 0}}{ }^{\mathbf{a}} \\
\mathbf{( m M )}\end{array}$ & $\begin{array}{c}\mathbf{C C}_{\mathbf{5 0}}^{\mathbf{b}} \\
\mathbf{( m M )}\end{array}$ \\
\hline Ligand-1 & 0.001 & 2.25 & 96 & 65.8 \\
\hline Ligand-2 & 0.598 & 2.67 & 29.3 & 110 \\
\hline Ligand-3 & 0.838 & 3.25 & 5.3 & 132 \\
\hline
\end{tabular}

Table 2: Bio-activity properties of different ligands.
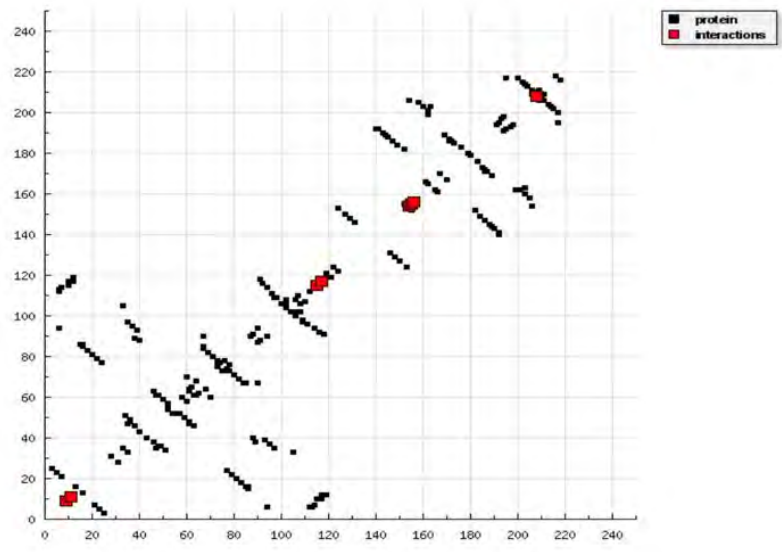

Figure 7: HB plot of ligand-3 with p24 HIV protein.

\section{Discussions}

Outcome of present research

From the present study, it is observed that molecules which have high dipole moments exhibit higher $\operatorname{LogP}$ and $\mathrm{CC}_{50}$ values

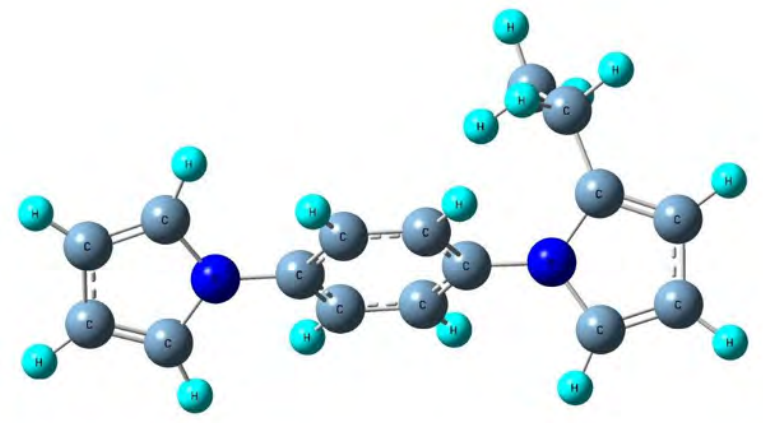

Figure 8: Optimized geometry of Ligand-3.

but lower $\mathrm{IC}_{50}$ values. It is known that in general, molecules having high hydrophobicity are less toxic. Present study is correlated with this fact. The LogP value of Ligand-3 (3.25) is the highest among the three ligands reported here. The $\mathrm{CC}_{50}$ value of this ligand (132) is the largest. It is also found that high dipole moment and $\log \mathrm{P}$ values are associated with low $\mathrm{IC}_{50}$ values which means they are highly active HIV-1 CA inhibitors. These results are obvious. But the most important observation of this study is that to improve the $\log \mathrm{P}$ and reduce the $\mathrm{IC}_{50}$ value of a compound requires the addition of hydrocarbon chain with proper increase of dipole moment.

\section{Comparison of different theoretical results}

It is very important to compare the $\mathrm{IC}_{50}$ values obtained from different theoretical models to endorse a model for future studies. Thus, we have presented the computed $\mathrm{IC}_{50}$ values obtained from different theoretical tools in table 3. From the presented values of $\mathrm{IC}_{50}$, it is observed that the order of RICM and QICM IC ${ }_{50}$ values obtained from RICM and QICM, are same as we obtained from the docking results though the values are not same. Quantum computed IC $_{50}$ values are lower compared to the respective docking $\mathrm{IC}_{50}$ values. Bag [33] has shown that quantum computation of $\mathrm{IC}_{50}$ produces better results compared to the experimented $\mathrm{IC}_{50}$ than the molecular docking method of IC $_{50}$ due to the inclusion of chemical behaviour of the inhibitors. Though the quantum computation method [34] is very accurate and we also did the molecular docking based $\mathrm{IC}_{50}$ computation of our compounds, still, we further compute $\mathrm{IC}_{50}$ of these compounds using the relative toxicity method [33] which are also 
presented in Table - 3, for the test of the newly reported approach of $\mathrm{IC}_{50}$ computation. For this calculation Cyclophiline $\mathrm{A}$ is used because, it is the substrate of this process. These $\mathrm{IC}_{50}$ values are higher compared to both molecular docking values and quantum computed values. But importantly, the order of $\mathrm{IC}_{50}$ values is same. It is mentioned that substrate based computation of relative $\mathrm{IC}_{50}$ is not accurate as it is for similar compound based computation [33]. In spite of this fact, we report these values to show that these compounds are really capable of inhibiting the substrate, Cyclophiline A.

\section{Comparison with previous results}

At present, state of the art HIV research focused on CA inhibition as this class of drugs are mutation registrant drugs. Presently a handful of HIV-1 CA inhibitors are reported [24-26,49]. Different ligands have preferences for different binding sites also. Detailed theoretical and experimental studied have been carried with these compounds. Their binding affinity range from $\mu \mathrm{M}$ to $\mathrm{nM}$. In another research work [50], we found that Ru5(CO)14( $\mu-\mathrm{H})$ $(\mu-\mathrm{S}(\mathrm{CH} 2) 2 \mathrm{COO}-) \mathrm{Na}+$ has the HIV-1 CA inhibition activity at the picomolar level. Still, we are promoting our present model ligands as HIV-1 CA inhibitor which has the activity at the micro molar level because, these are very common chemicals, very small in size, high binding frequency as well. Thus, these compounds would be cost-effective HIV drugs. In this regards, present work is very important and will be fruitful for future research.

\section{Conclusions}

In this research work small organic molecules are modelled and tested for HIV-1 Capsid-A inhibitor capacity. It is observed that 1,4-dicyclopentyl-2- ethylbenzene has very good inhibition constant (5.3 $\mu \mathrm{M}$ computed using quantum computation method). Though, several inhibitors of the same class with better activity is reported, this com-pound has the privilege in terms of production cost as it is a very small organic compound and shows very less toxicity with good hydrophobicity. Thus, this compound could be pursued for in-vitro test.

\section{Bibliography}

1. Ahmed Shaimaa M., et al. "Comparison of the molecular dynamics and calculated binding free energies for nine FDAapproved HIV-1 PR drugs against subtype B and C-SA HIV PR". Chemical Biology and Drug Design 81.2 (2013): 208-218.
2. Adamson Catherine S and Eric O Freed. "Anti-HIV-1 therapeutics: from FDA-approved drugs to hypothetical future targets". Molecular Interventions 9.2 (2009): 70.

3. Kinch Michael S and Eric Patridge. "An analysis of FDA-approved drugs for infectious disease: HIV/AIDS drugs". Drug Discovery Today 19.10 (2014): 1510-1513.

4. Ghosh Arun K., et al. "Syntheses of FDA approved HIV protease inhibitors”. Synthesis 2001.15 (2001): 2203.

5. Zdanowicz Martin M. "The pharmacology of HIV drug resistance". American Journal of Pharmaceutical Education 70.5 (2006).

6. Freed Eric 0. "HIV-1 gag proteins: diverse functions in the virus life cycle”. Virology 251.1 (1998): 1-15.

7. Reicin Alise S., et al. "The role of Gag in human immunodeficiency virus type 1 virion morphogenesis and early steps of the viral life cycle". Journal of virology 70.12 (1996): 86458652.

8. Kiernan Rosemary E., et al. "Role of matrix in an early postentry step in the human immunodeficiency virus type 1 life cycle". Journal of Virology 72.5 (1998): 4116-4126.

9. Freed, Eric 0. "HIV-1 replication". Somatic Cell and Molecular Genetics 26.1-6 (2001): 13-33.

10. Dragic Tatjana. "An overview of the determinants of CCR5 and CXCR4 co-receptor function". Journal of General Virology 82.8 (2001): 1807-1814.

11. Bour Stephane., et al. "The human immunodeficiency virus type 1 (HIV-1) CD4 receptor and its central role in promotion of HIV-1 infection". Microbiological Reviews 59.1 (1995): 6393.

12. Dunne Dana $W$ and Michael J Mugavero. "Topics in Antiviral Medicine $^{\mathrm{TM} ”} .24 .3$ (2016): 132.

13. Johnson Victoria A., et al. "Drug resistance mutations in HIV-1". 11.6 (2003): 215-221.

14. Delaugerre C., et al. "Resistance profile and cross-resistance of HIV-1 among patients failing a non-nucleoside reverse transcriptase inhibitor-containing regimen". Journal of Medical Virology 65.3 (2001): 445-448. 
15. Tang Chun., et al. "Antiviral inhibition of the HIV-1 capsid protein". Journal of Molecular Biology 327.5 (2003): 1013-1020.

16. Li Guangdi., et al. "Functional conservation of HIV-1 Gag: implications for rational drug design". Retrovirology 10.1 (2013): 126.

17. Gitti Rossitza K., et al. "Structure of the amino-terminal core domain of the HIV-1 capsid protein". Science 273.5272 (1996): 231-235.

18. Gamble Theresa R., et al. "Structure of the carboxyl-terminal dimerization domain of the HIV-1 capsid protein". Science 278.5339 (1997): 849-853.

19. Pornillos Owen., et al. "Atomic-level modelling of the HIV capsid". Nature 469.7330 (2011): 424-427.

20. Pornillos Owen, et al. "X-ray structures of the hexameric building block of the HIV capsid". Cell 137.7 (2009): 1282-1292.

21. Gres Anna T., et al. "X-ray crystal structures of native HIV1 capsid protein reveal conformational variability". Science 349.6243 (2015): 99-103.

22. Goudreau Nathalie., et al. "Novel inhibitor binding site discovery on HIV-1 capsid N-terminal domain by NMR and X-ray crystallography". ACS Chemical Biology 8.5 (2013): 1074-1082.

23. Lemke Christopher T., et al. "Distinct effects of two HIV-1 capsid assembly inhibitor families that bind the same site within the N-terminal domain of the viral CA protein". Journal of Virology 86.12 (2012): 6643-6655.

24. Sticht Jana., et al. "A peptide inhibitor of HIV-1 assembly in vitro". Nature Structural and Molecular Biology 12.8 (2005): 671-677.

25. Shi Jiong., et al. "Small-molecule inhibition of human immunodeficiency virus type 1 infection by virus capsid destabilization". Journal of Virology 85.1 (2011): 542-549.

26. Teeraananchai S., et al. "Life expectancy of HIV-positive people after starting combination antiretroviral therapy: a meta-analysis". HIV Medicine 18.4 (2017): 256-266.

27. Thangarasu P., et al. "Unveiling novel 2-cyclopropyl-3-ethynyl-4- (4-fluorophenyl) quinolines as GPCR ligands via PI3-kinase/PAR-1 antagonism and platelet aggregation valuations; development of a new class of anticancer drugs with thrombolytic effects". Bioorganic Chemistry 81 (2018): 468-480.

28. Roy Kunal., et al. A primer on QSAR/QSPR modeling: fundamental concepts. Springer (2015).

29. Bierbach Ulrich. "Annual Review of Biophysics and Biomolecular Structure, Volume 32 Edited by Robert M. Stroud (University of California, San Francisco), Wilma K. Olson (Rutgers University), and Michael P. Sheetz (Columbia University). Annual Reviews: Palo Alto, CA. 2003. xvi+ 534 pp. 80.00PrintVersionforIndividuals, 180 Print Version for Institutions. (2004): 4742-4743.

30. Shoichet Brian K., et al. "Molecular docking using shape descriptors". Journal of Computational Chemistry 13.3 (1992): 380-397.

31. Knegtel Ronald MA., et al. "Molecular docking to ensembles of protein structures". Journal of Molecular Biology 266.2 (1997): 424-440.

32. Bikadi Zsolt and Eszter Hazai. "Application of the PM6 semiempirical method to modeling proteins enhances docking accuracy of AutoDock". Journal of Cheminformatics 1.1 (2009): 15.

33. Bag Arijit. "DFT based computational methodology of IC50 prediction”. Current Computer-aided Drug Design (2020).

34. Bag Arijit and Pradip Kr Ghorai. "Development of quantum chemical method to calculate half maximal inhibitory concentration (IC50)". Molecular Informatics 35.5 (2016): 199-206.

35. Bag Arijit and Pradip Kr Ghorai. "Computational investigation of the ligand field effect to improve the photoacoustic properties of organometallic carbonyl clusters". RSC Advances 5.40 (2015): 31575-31583.

36. Perdew John P. “Density-functional approximation for the correlation energy of the inhomogeneous electron gas". Physical Review B 33.12 (1986): 8822.

37. Krim J., et al. "Triple-point wetting of light molecular gases on Au (111) surfaces". Physical Review Letters 52.8 (1984): 640.

38. Bultinck Patrick., et al. eds. Computational medicinal chemistry for drug discovery. CRC Press (2003). 
39. Tirado-Rives Julian and William L Jorgensen. "Performance of B3LYP density functional methods for a large set of organic molecules". Journal of Chemical Theory and Computation 4.2 (2008): 297-306.

40. Wang Nick X and Angela K Wilson. "Effects of basis set choice upon the atomization energy of the second-row compounds SO2, $\mathrm{CCl}$, and $\mathrm{ClO} 2$ for B3LYP and B3PW91". The Journal of Physical Chemistry A 107.34 (2003): 6720-6724.

41. Frisch A. "Gaussian” Wallingford, USA, 25 (2009).

42. Bode Brett M and Mark S Gordon. "MacMolPlt: a graphical user interface for GAMESS". Journal of Molecular Graphics and Modelling 16.3 (1998): 133-138.

43. Kong Jing., et al. "Q-Chem 2.0: a high-performance ab initio electronic structure program package". Journal of Computational Chemistry 21.16 (2000): 1532-1548.

44. Giannozzi Paolo., et al. "QUANTUM ESPRESSO: a modular and open-source software project for quantum simulations of materials". Journal of Physics: Condensed Matter 21.39 (2009): 395502.

45. Hafner Jürgen. "Ab-initio simulations of materials using VASP: Density-functional theory and beyond". Journal of Computational Chemistry 29.13 (2008): 2044-2078.

46. Kendall Ricky A., et al. "High performance computational chemistry: An overview of NWChem a distributed parallel application". Computer Physics Communications 128.1-2 (2000): 260-283.

47. Lipinski Christopher A., et al. "Experimental and computational approaches to estimate solubility and permeability in drug discovery and development settings". Advanced Drug Delivery Reviews 23.1-3 (1997): 3-25.

48. Ishihara Mariko., et al. "Estimation of relationship between descriptors and cytotoxicity of newly synthesized 1, 2, 3, 4-tetrahydroisoquinoline derivatives". Anticancer Research 29.10 (2009): 4077-4082.

49. Singh Kamlendra., et al. "GS-CA compounds: first-in-class HIV1 capsid inhibitors covering multiple grounds". Frontiers in Microbiology 10 (2019): 1227.

50. Bag Arijit. "In Search of Multi-Stage Mutation Resistant HIV Drugs: A DFT Study" (3/31/2020).

\section{Assets from publication with us}

- Prompt Acknowledgement after receiving the article

- Thorough Double blinded peer review

- Rapid Publication

- Issue of Publication Certificate

- High visibility of your Published work

Website: www.actascientific.com/

Submit Article: www.actascientific.com/submission.php

Email us: editor@actascientific.com

Contact us: +919182824667 